\title{
Efficient reevaluation of surface displacements in a layered elastic half-space
}

\author{
Andersen, Sebastian; Levenberg, Eyal; Andersen, Mathias B.
}

Published in:

The International Journal of Pavement Engineering

Link to article, DOI:

$10.1080 / 10298436.2018 .1483502$

Publication date:

2020

Document Version

Peer reviewed version

Link back to DTU Orbit

Citation (APA):

Andersen, S., Levenberg, E., \& Andersen, M. B. (2020). Efficient reevaluation of surface displacements in a layered elastic half-space. The International Journal of Pavement Engineering, 21(4), 408-415.

https://doi.org/10.1080/10298436.2018.1483502

\section{General rights}

Copyright and moral rights for the publications made accessible in the public portal are retained by the authors and/or other copyright owners and it is a condition of accessing publications that users recognise and abide by the legal requirements associated with these rights.

- Users may download and print one copy of any publication from the public portal for the purpose of private study or research.

- You may not further distribute the material or use it for any profit-making activity or commercial gain

- You may freely distribute the URL identifying the publication in the public portal

If you believe that this document breaches copyright please contact us providing details, and we will remove access to the work immediately and investigate your claim. 


\section{Efficient Reevaluation of Surface Displacements in a Layered Elastic Half-space}

In situ evaluation of mechanical pavement properties requires fitting measured surface displacements with model displacements. Such inverse analysis is guided by optimization algorithms that entail re-execution of the underlying model many times over. For layered elasticity, which is the most commonly employed pavement model, this involves calculating computationally demanding semiinfinite integrals in every optimization step. In this connection, a method was proposed to improve the computational efficiency of surface displacement recalculations in layered elastic systems. It was based on manipulating the original integrals by adding and then subtracting carefully selected auxiliary functions so that they remained mathematically unchanged, yet became faster to compute. The auxiliary functions were derived from an analytic interrogation, rendering the formulation robust and applicable to any layered elastic system without practical restrictions on the model parameters. Overall, the method is deemed consistently more efficient than standard evaluation techniques for achieving a given accuracy level.

Keywords: Layered elastic theory, Computational efficiency, Surface displacements, Numerical integration, Inverse analysis

\section{Introduction}

Estimating the mechanical properties of pavements is a routine non-destructive activity, typically carried out by in situ deflection testing, followed by in silico inverse analysis. The standard device for in situ testing is the falling weight deflectometer (ASTM 2015a, 2015b); while stationary, it applies a load at the surface by dropping a mass, and concurrently records vertical surface displacements at several offset distances. In recent years the engineering community has shown increasing interest in developing alternative pavement evaluation devices that perform measurements while moving (Andersen et al. 2017, Flintsch et al. 2013, Rada et al. 2015). These modern devices are essentially custom-built trucks, heavily instrumented with sophisticated sensory gear, expected to traverse pavement networks at highway speeds and record surface displacements generated by their own presence. 
The usual approach for performing the inverse analysis involves mechanical modeling of the pavement system, forward-simulating the applied loading and resulting displacements, and then identifying the layer properties by best fitting simulated displacements with their respective measurements (e.g., Madsen and Levenberg 2017). This process is guided by optimization algorithms that require re-execution of the forward simulation many times over with different combinations of layer properties. Accurate displacement evaluation in the forward simulation is obligatory for navigating towards convergence because calculations must exhibit correct sensitivity to small changes in layer properties; this feature is especially important when gradient-based optimization techniques are employed. It is also noted that the accuracy level of model displacements should be superior to the experimental data (Liu and Han 2003). Concurrently, displacement calculations should be carried out efficiently because the entire process of identifying layer properties must be completed within a practical time interval. Considering that the new devices collect huge amounts of displacement data for subsequent interpretation, the demand for computational efficiency becomes critical.

Within the engineering community the most common mechanical model employed for representing pavement systems is layered elasticity (AASHTO 2008, Ullidtz et al. 2010, Zhou et al. 2010). While the underlying formulation is analytic, numerical evaluation of semi-infinite integrals is required, involving integrands that are both oscillating and computationally expensive. In the particular case of calculating surface responses, the integrals exhibit slow convergence and the computational costs are highest. This issue is the main reason why approximate theories with closed form algebraic expressions are still being used (Dalla Valle and Thom 2016, Ullidtz 1987).

Improving the evaluation accuracy and efficiency of layered elastic surface solutions has been addressed in several studies. Chen (1971) proposed a method to circumvent the numerical difficulty in convergence of stresses and displacements by subtracting a half-space formulation before performing the integration, and then adding the half-space solution to the computed results. This approach can be dated back to a classic numerical technique known as 'singularity subtraction' (Abramowitz 1954). Chen did not quantify the gained computational efficiency of his method. Maina and Matsui (2005) tackled the semi-infinite integrals by means of double-exponential quadrature combined with Richardson's extrapolation (Sugihara, 1987). Also in this case, no quantified information was provided on the gained computational efficiency. 
Pan et al. (2007) proposed an algorithm for rapid calculation of surface displacements due to a uniform circular pressure load on the surface of the layered elastic half-space. This was done based on a two-stage approach. First, for a limited number of radial distances, surface displacements were solved using a highly accurate yet computationally expensive method that combined the Lucas algorithm (Lucas 1995, Lucas and Stone 1995) with adaptive Gauss quadrature. Next, the accurate solutions were interpolated in order to manufacture displacements for many intervening locations at a much lower computational cost. Essentially, the investigation focused on correctly choosing the spacing of the accurate solutions and on appropriately selecting an interpolation scheme - and not on improving the efficiency of the underlying calculation kernel.

In the work of Khazanovich and Wang (2007) two improvement measures were suggested to expedite layered elastic calculations for surface points. The first was aimed at lowering the computational cost involved in reevaluating the integrands at the expense of confining the solution correctness to a certain family of layered elastic problems. This was achieved by means of spline approximation, after collocating the integrands at some preselected key points. Guidance on how to choose these key spline knots was not provided. The second improvement measure included the half-space 'subtraction and addition' technique suggested by Chen (1971). The combined effect of both measures was demonstrated to yield improved accuracy and reduced calculation time in the order of $75 \%$ compared to WinJulea, a publically available program by US Army Corps of Engineers (2010).

Wang et al. (2010) offered a different, non-traditional, derivation to evaluate responses in layered elastic systems. Their approach employed a recurrence relationship (in Hankel transformed domain) between surface responses and internal points. Though not demonstrated, the authors claimed that their derivation provides a more rapid solution time than standard approaches. Erlingsson and Ahmed (2013) described the development of a fast layered elastic code. Their work commenced with a literature survey of relevant techniques, and concluded with implementing double-exponential quadrature and Richardson's extrapolation as suggested in Maina and Matsui (2005) combined with spline approximation as suggested in Khazanovich and Wang (2007). Also in this case the chosen spline knots were not disclosed. By comparing against WinJulea, reduction in calculation time of the order of $30 \%$ was demonstrated. 
In a recent contribution, Zhao et al. (2015) attempted to improve near-surface layered elastic calculations, mainly in terms of accuracy, but also in terms of calculation speed. To achieve this, they split the semi-infinite integral into a sum of: (i) a definite integral evaluated with a standard quadrature approach, and (ii) a 'tail integral' extending to infinity, the integrand of which assumed to closely match its asymptotic behavior. This latter semi-infinite integral was evaluated by the technique suggested in Lucas and Stone (1995) wherein the integrand is decomposed into a sum of two damped sinusoidal functions that may be accurately integrated with classic sequence acceleration approaches. By comparison to other programs the authors demonstrated superior accuracy for surface responses, yet slower calculations (as also noticed and reported in Pan et al. (2007)). Faster calculations were achieved only after including the spline approximation suggested in Khazanovich and Wang (2007).

This paper contributes to the body of work dealing with expediting layered elastic calculations. It proposes a new method for efficient reevaluation of surface displacements useful for optimization processes. The method does not involve spline approximation (i.e., integrand interpolation) nor does it entail any kind of extrapolation techniques, series acceleration or asymptotic representation. In turn, it is based on generalizing and further improving the 'subtraction and addition' technique suggested in Chen (1971) and in Khazanovich and Wang (2007). The development is specifically tailored for inverse analysis situations, wherein system responses at several predefined locations must be repeatedly evaluated with new layer properties. The paper commences by restating the standard layered elastic formulation for evaluating surface displacements. It then identifies and illustrates where high calculation costs reside. The new proposed method is presented and explained next, followed by a demonstration for a typical pavement system. The paper concludes with a short discussion on the merits of the proposed approach, and lists some potential ideas for further upgrading.

\section{General Formulation}

With reference to Figure 1, a stratified half-space is considered, consisting of $N$ horizontal layers each linear elastic, isotropic, homogeneous, and weightless. A cylindrical coordinate system is located at the top boundary with the radial coordinate $r$ coinciding with the surface and the vertical coordinate $z$ directed into the medium. The material properties of layer $n \in[1, N]$ are Young's modulus $E_{n}$ and Poisson's ratio $v_{n}$; 
the distance from the surface to the bottom of the $n^{\text {th }}$ layer is $z_{n}$, with $z_{N-1}=H$, and $z_{N}=\infty$. Furthermore, a circular distributed load with radius $a$ and intensity $q$ is applied to the surface with its center at $r=0$. The vertical and radial displacements of any point in the medium are referred to as $u_{z}=u_{z}(z, r)$ and $u_{r}=u_{r}(z, r)$ respectively.

The $N$-layered elastic solution for the displacements at the surface $(z=0)$ in the vertical direction and in the radial direction can be mathematically expressed as (e.g., Huang 2004)

$$
\begin{aligned}
& u_{z}(0, r)=C_{z} \int_{0}^{\infty} O_{z}(m, r) F_{z} d m \\
& u_{r}(0, r)=C_{r} \int_{0}^{\infty} O_{r}(m, r) F_{r} d m
\end{aligned}
$$

wherein $C_{z}=-q \alpha H\left(1+v_{1}\right) / E_{1}$ and $C_{r}=-C_{z}$ are constants (units of length), $\alpha=a / H$ is a normalized radius of loaded area, and $O_{z}(m, r)=J_{0}(m \rho) J_{1}(m \alpha) / m$ and $O_{r}(m, r)=J_{1}(m \rho) J_{1}(m \alpha) / m$ are unitless decaying oscillatory functions. The explicit dependence of $O_{z}(m, r)$ and $O_{r}(m, r)$ on $a$ is not indicated because the loaded radius is usually a known parameter when performing inverse analysis. Furthermore, $J_{0}(\cdot)$ and $J_{1}(\cdot)$ are zero and first order Bessel functions of the first kind, $m$ is a unitless integration parameter, and $\rho=r / H$ represents a dimensionless radial coordinate indicating the displacement evaluation location. For a given $\alpha$ these oscillatory functions decay towards zero at an asymptotic rate of about $m^{-2} \rho^{-0.5}$. The functions $F_{z}=F_{z}\left(m, E_{n}, v_{n}, z_{n}\right)$ and $F_{r}=F_{r}\left(m, E_{n}, v_{n}, z_{n}\right)$ are obtained from solving a linear set of $4 N-2$ coupled equations that represent the problem boundary conditions; as indicated, each of them depends on the integration parameter $m$ as well as on the full set of system characteristics, i.e., Young's moduli $E_{n}$, Poisson's ratios $v_{n}$, and layer interface depths $z_{n}$ 


\section{Standard Evaluation Method}

The surface displacements according to Equations (1) and (2) are evaluated by numerical integration. For this purpose, the semi-infinite integrals are typically partitioned into a finite sequence of definite integrals; the partitioning is chosen such that each finite integral is evaluated over an interval covering two consecutive zeros of the integrands (Cornille 1972, Hillion and Nurdin 1977, Longman 1956). The functions $F_{z}$ and $F_{r}$ are well behaved and do not change sign for most cases of practical relevance; therefore, they are not considered in the partitioning. Thus, the integrand zero points are essentially dictated by the oscillatory functions. Defining $K_{z}$ and $K_{r}$ as the total number of integral partitions, the surface displacements are approximated as

$$
\begin{aligned}
& u_{z}^{0}\left(K_{z}\right)=C_{z} \sum_{k=1}^{K_{z}} \int_{m_{z, k}}^{m_{z, k+1}} O_{z}(m, r) F_{z} d m \\
& u_{r}^{0}\left(K_{r}\right)=C_{r} \sum_{k=1}^{K_{r}} \int_{m_{r, k}}^{m_{r, k+1}} O_{r}(m, r) F_{r} d m
\end{aligned}
$$

where $u_{z}^{0}\left(K_{z}\right) \rightarrow u_{z}(0, r)$ as $K_{z} \rightarrow \infty$ and $u_{r}^{0}\left(K_{r}\right) \rightarrow u_{r}(0, r)$ as $K_{r} \rightarrow \infty$. The parameter $m_{z, k}$ represents the $k^{\text {th }} m$-value for which $O_{z}(m, r)$ is zero, i.e., either $J_{0}(m \rho)$ or $J_{1}(m \alpha)$ are zero. Similarly, $m_{r, k}$ represents the $k^{\text {th }} m$-value for which $O_{r}(m, r)$ is zero, i.e., either $J_{1}(m \rho)$ or $J_{1}(m \alpha)$ are zero. Furthermore, $\Delta m_{z, k}=m_{z, k+1}-m_{z, k}$ and $\Delta m_{r, k}=m_{r, k+1}-m_{r, k}$ indicate the span of the $k^{\text {th }}$ integration interval. The first zero points of $O_{z}(m, r)$ and $O_{r}(m, r)$ are at $m=0$; thus, $m_{z, 1}=0$ and $m_{r, 1}=0$, and the lower integration limits in Equations (3) and (4) correspond to those in Equations (1) and (2).

For a given layered system with a given load radius, and for a given radial coordinate, heavy computational cost may be associated with evaluating Equations (3) and (4) to a high degree of accuracy. This cost is attributed to the slow convergence of the sequences in combination with the effort needed for evaluating $F_{z}$ and $F_{r}$ given that both functions require inverting a new $(4 N-2) \times(4 N-2)$ matrix at every integration point $m$. As the integral partitioning depends on the radial coordinate, a different set of integration points is employed in Equations (3) and (4) when a different deformation location is evaluated. This means that the functions $F_{z}$ and $F_{r}$ must be recalculated for 
each considered point, even though $E_{n}, v_{n}$ and $a$ are unchanged. Typically, a Gauss quadrature method, either adaptive (Pan 1997, Pan et al. 2007), or with a preselected number of nodes (Chen 1971, Cornille 1972, Hillion and Nurdin 1977, Huang 2004, Longman 1956, Peutz et al. 1968) is preferred for evaluating the definite integrals. Therefore, in either case, it is the number of chosen partitions, i.e., the values of $K_{z}$ and $K_{r}$, that essentially govern the overall computational effort and associated solution accuracy.

\section{Problem Exemplification}

For demonstrating the inefficiency of the standard evaluation method, a typical system consisting of four layers with a single surface load is hereafter employed as example. Layer thicknesses and elastic properties are listed in Table 1; the loading conditions are $a=140 \mathrm{~mm}$ and $q=0.7 \mathrm{MPa}$.

First evaluated were ultra-accurate vertical and radial surface displacements $u_{z, \text { ref }}^{0}$ and $u_{r, \text { ref }}^{0}$. These were computed at a high computational cost involving a very large number of partitions $K_{z}=K_{r}=1000$ and a 30-point Gauss formula for the definite integrals. Radial offsets in the range of $r=a^{+}$to $r=25 a$ were considered for analysis; this choice represents a realistic sensing range for modern deflection measuring devices. In particular (for the considered case), $r=a^{+}$designates a point just outside a tirepavement contact zone whereas $r=25 a$ designates a point with an offset of $3.5 \mathrm{~m}$. The outcome of these calculations served as error reference for other displacement calculations involving much reduced computational effort of $K_{z}, K_{r} \in[1,100]$. The errors were defined as

$$
\begin{aligned}
& \operatorname{Err}_{z}\left(K_{z}\right)=\left|\frac{u_{z}^{0}\left(K_{z}\right)-u_{z, \text { ref }}^{0}}{u_{z, \text { ref }}^{0}}\right| \\
& \operatorname{Err}_{r}\left(K_{r}\right)=\left|\frac{u_{r}^{0}\left(K_{r}\right)-u_{r, \text { ref }}^{0}}{u_{r, \text { ref }}^{0}}\right|
\end{aligned}
$$


wherein $\operatorname{Err}_{z}\left(K_{z}\right)$ represents the absolute error in $u_{z}^{0}\left(K_{z}\right)$ relative to $u_{z \text {,ref }}^{0}$, and $\operatorname{Err}_{r}\left(K_{r}\right)$ represents the absolute error in $u_{r}^{0}\left(K_{r}\right)$ relative to $u_{r, \text { ref }}^{0}$.

In Figure 2, these error terms are plotted in log scale versus the number of partitions. Two separate charts are included, one for $\operatorname{Err}_{z}\left(K_{z}\right)$ (Figure 2a) and another for $\operatorname{Err}_{r}\left(K_{r}\right)$ (Figure 2b). Discrete $K_{z}$ and $K_{r}$ values were employed for the calculations, and the resulting points were converted into continuous lines for graphical clarity. Only three offset radii are displayed in the figure, the edge cases $r=a^{+}$and $r=25 a$, and an intermediate value $r=5 a$. Calculation results are depicted with thin grey lines, whereas thicker black lines represent upper error envelopes - introduced to help visualize the overall behavior. As can be seen in both charts, the errors are oscillatory, yet with an overall decreasing trend emphasized by the error envelopes. The oscillatory behavior is a direct result of choosing to integrate between the zeroes of the Bessel functions. More specifically, this behavior originates from repeated sign changes of the definite integrals in combination with an uneven distribution of integration interval sizes $\Delta m_{z, k}$ and $\Delta m_{r, k}$ . Due to the logarithmic scale in the charts the oscillating error amplitudes for $r=5 a$ and $r=25 a$ appear to be of constant magnitude or slightly increasing; in effect, they are all decreasing. It may also be seen that errors depend on the specific displacement (either $u_{z}^{0}$ or $u_{r}^{0}$ ) and on the offset radius $r$. For the given case, radial displacements $u_{r}^{0}$ appear more computationally demanding compared to vertical displacements $u_{z}^{0}$. Figure 2 also shows that evaluating all surface displacements (within the chosen analysis range of $a^{+} \leq r \leq 25 a$ ) with an accuracy level of at least $0.1 \%$ requires up to about 90 integration partitions.

Based on the aforementioned observations it becomes clear why the effort for computing surface displacements is high, nearly prohibitive, for suiting the needs of modern deflection-measuring devices. Assuming a 10-point Gauss formula for evaluating the definite integrals, in combination with 20 partitions for attaining a sufficiently accurate displacement solution, about 400 matrix inversions are needed for evaluating both $u_{z}^{0}$ and $u_{r}^{0}$ at a particular radial location, and for a given set of trial elastic moduli. Moreover, the new devices are expected to sample pavement displacements simultaneously at about 10 different radial locations (Pedersen et al. 2013), with an acquisition rate of the order of $1000 \mathrm{~Hz}$ (Jack Larsen, R\&D Manager at Dynatest 
International, personal communication, 31 January 2018). Hence, about 20,000 measured displacement values will be collected for subsequent inverse analysis every second. Even if some averaging techniques are employed to smooth the data and a priori reduce interpretation demands, there is a clear incentive for improving computational effectiveness.

\section{Proposed Method}

The approach herein proposed for improving the reevaluation efficiency of $u_{z}^{0}$ and $u_{r}^{0}$ builds on the ideas suggested in Chen (1971) and Khazanovich and Wang (2007). In analogy to Equations (1) or (2), consider the task of integrating over a semi-infinite range the function $f=f(m)$ which oscillates and decays slowly towards zero as $m$ increases towards infinity. Instead of directly partitioning this integral $(I)$ for subsequent evaluation as a sequence, similar to Equations (3) and (4), it is first decomposed into a sum of two integrals $I=I_{1}+I_{0}$ as follows

$$
\int_{0}^{\infty} f d m=\underbrace{\int_{0}^{\infty}(f-g) d m}_{I_{1}=I-I_{0}}+\int_{0}^{\infty} g d m
$$

wherein $g=g(m)$ is some user-defined auxiliary function. From a mathematical viewpoint, the evaluation of $I_{1}+I_{0}$ is equivalent to the evaluation of $I$ because the influence of $g$ is subtracted and then added, and therefore cancels out. However, from a computational perspective, the evaluation of $I_{1}+I_{0}$ is not equivalent to the evaluation of $I$. Specifically, the function $g$ can be chosen to oscillate similar to $f$, and at the same time produce an integrand $f-g$ that decays faster (compared to $f$ ) towards zero as $m$ increases. This means that numerical evaluation of $I_{1}$ requires a shorter evaluation sequence (i.e., less partitions), and therefore becomes more computationally efficient than $I$. Of course, for this to be justified, the auxiliary function $g$ should also be chosen such that the integral $I_{0}$ is computationally cheap to evaluate or to reevaluate when the problem parameters change.

The above formulation is applied to the problem at hand, separately for the vertical and radial directions. For this purpose, the following auxiliary functions $g_{z}$ and $g_{r}$ are recommended for the vertical and radial directions 


$$
\begin{gathered}
g_{z}=O_{z}(m, r) W_{z} \\
g_{r}=O_{r}(m, r) W_{r}
\end{gathered}
$$

wherein $W_{z}$ and $W_{r}$ are non-oscillatory expressions of $m$ that are less expensive to evaluate compared with $F_{z}$ and $F_{r}$. Hereafter, these expressions are referred to as 'Accelerators', because they serve the purpose of speeding up the rate of integration convergence. With these definitions, the integrals in Equations (1) and (2) become

$$
\begin{aligned}
& \underbrace{u_{z}(0, r)}_{I_{z}}=\underbrace{C_{z} \int_{0}^{\infty} O_{z}(m, r)\left(F_{z}-W_{z}\right) d m}_{I_{z 1}=I_{z}-I_{z 0}}+\underbrace{C_{z} \int_{0}^{\infty} g_{z} d m}_{I_{z 0}} \\
& \underbrace{u_{r}(0, z)}_{I_{r}}=\underbrace{C_{r} \int_{0}^{\infty} O_{r}(m, r)\left(F_{r}-W_{r}\right) d m}_{I_{r 1}=I_{r}-I_{r 0}}+\underbrace{C_{r} \int_{0}^{\infty} g_{r} d m}_{I_{r 0}}
\end{aligned}
$$

from which it is self-evident that the Accelerators should be chosen to match, as close as possible, the functions $F_{z}$ and $F_{r}$, especially for large $m$ values. By so doing the integrals $I_{z 1}$ and $I_{r 1}$ will rapidly tend to zero, making their evaluation as a finite sequence both speedy and exact.

Subsequently, the asymptotic behavior of the functions $F_{z}$ and $F_{r}$ as $m \rightarrow \infty$ was investigated. This was performed analytically with a computer algebra platform, for an arbitrary system consisting of any number of layers and unspecified elastic properties. This investigation revealed that as $m$ increases towards infinity both functions approach constant values that only depend on the Poisson's ratio of the top layer

$$
\begin{aligned}
& F_{z}^{\infty}=\lim _{m \rightarrow \infty} F_{z}=2 v_{1}-2 \\
& F_{r}^{\infty}=\lim _{m \rightarrow \infty} F_{r}=2 v_{1}-1
\end{aligned}
$$


With a physically valid range of $0<v_{1}<0.5$, the relevant bounds are $-2<F_{z}^{\infty}<-1$ and $-1<F_{r}^{\infty}<0$. Additionally, by means of asymptotic analysis the analytic investigations uncovered that both $F_{z}^{\infty}$ and $F_{r}^{\infty}$ are always approached at a rate proportional to $e^{-2 m z_{1} / H}$ , i.e., the ratio $z_{1} / H$ influences the approach rate. Based on these observations a logical choice for the Accelerators is

$$
\begin{aligned}
& W_{z}=\frac{a_{z} m^{2}+b_{z} m+c_{z}}{e^{2 m z_{1} / H}}+F_{z}^{\infty} \\
& W_{r}=\frac{a_{r} m^{2}+b_{r} m+c_{r}}{e^{2 m z_{1} / H}}+F_{r}^{\infty}
\end{aligned}
$$

wherein the limit behavior and limit values of $W_{z}$ and $W_{r}$ as $m \rightarrow \infty$ are a priori assured to correspond to those of $F_{z}$ and $F_{r}$. Moreover, polynomial expressions are added in the numerators, with constants $a_{j}, b_{j}$ and $c_{j}(j \in[r, \mathrm{z}])$, to provide mathematical flexibility in matching this asymptotic behavior for any layered elastic case. It is suggested that these constants are evaluated by collocating $F_{z}$ at three preselected $m$ values $\left(m_{1}, m_{2}, m_{3}\right)$ and by collocating $F_{r}$ at three (other) preselected $m$ values $\left(m_{4}, m_{5}, m_{6}\right)$. This should be done for the particular system and evaluation point being considered, and requires solving two sets of three equations organized in matrix form as

$$
\begin{aligned}
& {\left[\begin{array}{lll}
m_{1}^{2} & m_{1} & 1 \\
m_{2}^{2} & m_{2} & 1 \\
m_{3}^{2} & m_{3} & 1
\end{array}\right]\left\{\begin{array}{l}
a_{z} \\
b_{z} \\
c_{z}
\end{array}\right\}=\left\{\begin{array}{l}
\left(F_{z}\left(m_{1}, \ldots\right)-F_{z}^{\infty}\right) e^{2 m_{1} z_{1} / H} \\
\left(F_{z}\left(m_{2}, \ldots\right)-F_{z}^{\infty}\right) e^{2 m_{2} z_{1} / H} \\
\left(F_{z}\left(m_{3}, \ldots\right)-F_{z}^{\infty}\right) e^{2 m_{3} z_{1} / H}
\end{array}\right\}} \\
& {\left[\begin{array}{lll}
m_{4}^{2} & m_{4} & 1 \\
m_{5}^{2} & m_{5} & 1 \\
m_{6}^{2} & m_{6} & 1
\end{array}\right]\left\{\begin{array}{l}
a_{r} \\
b_{r} \\
c_{r}
\end{array}\right\}=\left\{\begin{array}{l}
\left(F_{r}\left(m_{4}, \ldots\right)-F_{r}^{\infty}\right) e^{2 m_{4} z_{1} / H} \\
\left(F_{r}\left(m_{5}, \ldots\right)-F_{r}^{\infty}\right) e^{2 m_{5} z_{1} / H} \\
\left(F_{r}\left(m_{6}, \ldots\right)-F_{r}^{\infty}\right) e^{2 m_{6} z_{1} / H}
\end{array}\right\}}
\end{aligned}
$$


wherein the matrices on the left-hand side have a simple predefined inversion form. The particular $m$ values for the collocation are chosen where $F_{z}$ and $F_{r}$ are close to their asymptotic values. They are extracted from the expressions

$$
\begin{aligned}
& \frac{1}{e^{2 m_{i} z_{1} / H}}+F_{z}^{\infty}=\beta_{i} F_{z}^{\infty} \quad(i=1,2,3) \\
& \frac{1}{e^{2 m_{i} z_{1} / H}}+F_{r}^{\infty}=\beta_{i} F_{r}^{\infty} \quad(i=4,5,6)
\end{aligned}
$$

wherein $\beta_{1}=\beta_{4}=99.90 \%, \beta_{2}=\beta_{5}=99.95 \%$, and $\beta_{3}=\beta_{6}=99.99 \%$. These $\beta$ 's indicate desired closeness levels to the asymptotic values $F_{z}^{\infty}$ and $F_{r}^{\infty}$. After the collocation according to Equations (16) and (17), the resulting $m$ values indicate points where $W_{z}=F_{z}$ and $W_{r}=F_{r}$. Based on the above choice of $\beta$ 's the associated collocation points represent practical upper bounds for the integrals $I_{z 1}$ and $I_{r 1}$ in Equations (10) and (11) given that the integrands are nearly zero for larger $m$ values.

After extracting $m_{1}, \ldots, m_{6}$ from Equations (18) and (19), and after calculating $a_{j}$ ,$b_{j}$ and $c_{j}$ with Equations (16) and (17), the displacements in Equations (10) and (11) are approximated as, cf. Equations (3) and (4)

$$
\begin{aligned}
& u_{z}^{0}\left(K_{z}\right)=C_{z} \sum_{k=1}^{K_{z}} \int_{m_{z, k}}^{m_{z, k+1}} O_{z}(m, r)\left(F_{z}-W_{z}\right) d m+I_{z 0} \\
& u_{r}^{0}\left(K_{r}\right)=C_{r} \sum_{k=1}^{K_{r}} \int_{m_{r, k}}^{m_{r, k+1}} O_{r}(m, r)\left(F_{r}-W_{r}\right) d m+I_{r 0}
\end{aligned}
$$

The terms $I_{z 0}$ and $I_{r 0}$ are given by

$$
\begin{aligned}
& I_{z 0}=C_{z}\left(a_{z} I_{z}^{(1)}+b_{z} I_{z}^{(2)}+c_{z} I_{z}^{(3)}+F_{z}^{\infty} I_{z}^{(4)}\right) \\
& I_{r 0}=C_{r}\left(a_{r} I_{r}^{(1)}+b_{r} I_{r}^{(2)}+c_{r} I_{r}^{(3)}+F_{r}^{\infty} I_{r}^{(4)}\right)
\end{aligned}
$$

with $I_{z}^{(i)}$ and $I_{r}^{(i)}(i=1, \ldots, 4)$ as the following set of semi-infinite integrals 


$$
\begin{array}{ll}
I_{z}^{(1)}=\int_{0}^{\infty} \frac{m^{2} O_{z}(m, r)}{e^{2 m z_{1} / H}} d m & I_{r}^{(1)}=\int_{0}^{\infty} \frac{m^{2} O_{r}(m, r)}{e^{2 m z_{1} / H}} d m \\
I_{z}^{(2)}=\int_{0}^{\infty} \frac{m O_{z}(m, r)}{e^{2 m z_{1} / H}} d m & I_{r}^{(2)}=\int_{0}^{\infty} \frac{m O_{r}(m, r)}{e^{2 m z_{1} / H}} d m \\
I_{z}^{(3)}=\int_{0}^{\infty} \frac{O_{z}(m, r)}{e^{2 m z_{1} / H}} d m & I_{r}^{(3)}=\int_{0}^{\infty} \frac{O_{r}(m, r)}{e^{2 m z_{1} / H}} d m \\
I_{z}^{(4)}=\int_{0}^{\infty} O_{z}(m, r) d m & I_{r}^{(4)}=\int_{0}^{\infty} O_{r}(m, r) d m
\end{array}
$$

The above integrals are independent of layer properties and therefore need to be numerically evaluated only once for a given radial coordinate. This can be done e.g. by the standard method described previously. Thus, for an inverse analysis situation where $I_{z 0}$ and $I_{r 0}$ are evaluated a priori, the numerical cost of recalculating $u_{z}^{0}$ and $u_{r}^{0}$ in Equations (20) and (21) is essentially the same as the cost required to recalculate the sequences only. Therefore, in these situations the associated $K_{z}$ and $K_{r}$ represent the computational cost of the displacement formulations.

It should be noted that a 'special case' of the formulation exits, wherein zero polynomials are employed in Equations (14) and (15) (i.e., $a_{j}, b_{j}$ and $c_{j}$ are zero). This zero-setting saves the computational cost involved in six extra matrix inversions required by Equations (16) and (17) for evaluating $F_{z}$ and $F_{r}$ at the collocation points. Moreover, so doing annuls the need for evaluating the integrals in Equations (24)-(27). This special case also produces improved efficiency compared to the standard evaluation method in Equations (3) and (4). It is essentially equivalent to the formulation suggested in Chen (1971) and in Khazanovich and Wang (2007).

\section{Efficiency Analysis}

The newly proposed method offers considerable gains in numerical efficiency when reevaluating surface displacements, i.e., in cases where $I_{z 0}$ and $I_{r 0}$ can be evaluated a 
priori and therefore do not add to the computational cost. To demonstrate this, the pavement example in Table 1 is revisited - loaded vertically over a circular area with $a=140 \mathrm{~mm}$ and $q=0.7 \mathrm{MPa}$. The vertical and radial displacements are evaluated at three radial offsets: $r=a^{+}, r=5 a$ and $r=25 a$. Results are presented in Figure 3 for the vertical displacements and in Figure 4 for the radial displacements. Similar to Figure 2 they depict displacement accuracy, considering the error definitions in Equations (5) and (6), versus computational cost, i.e., the number of integration partitions $K_{z}$ and $K_{r}$. Each figure contains three charts, each referring to the three different radial offsets: $r=a^{+}$ (Figures $3 \mathrm{a}$ and $4 \mathrm{a}$ ), $r=5 a$ (Figures $3 \mathrm{~b}$ and $4 \mathrm{~b}$ ), and $r=25 \mathrm{a}$ (Figures $3 \mathrm{c}$ and $4 \mathrm{c}$ ).

The efficiency of the proposed method, Equations (20) and (21), is depicted with solid lines. Also shown are the special case results of the proposed formulation (dashed lines), wherein zero polynomials are employed in Equations (14) and (15). For graphical comparison purposes the relevant results from Figure 2 are replotted in Figures 3 and 4 (dash-dotted lines), contrasting the numerical inefficiency of the standard evaluation method, Equations (3) and (4), against the proposed method. Similar to Figure 2, the thin grey lines represent actual calculation results, whereas the thicker black lines represent upper error envelopes. As can be seen from all of the charts in both figures, the error of the proposed method dives very rapidly to negligible levels compared to the rate of error decrease of the standard method; the proposed method also displays superiority compared to the special case. A separate investigation showed that the proposed method exhibits superior efficiency as long as $z_{1} / H>1.5 \%$. For realistic pavement systems, wherein $H$ rarely exceeds $2 \mathrm{~m}$, this ratio translates into $z_{1}>30 \mathrm{~mm}$. Hence, all cases of practical relevance are covered.

Another graphical depiction of the computational advantage of the proposed method, based on the example case in Table 1, is offered in Figure 5. This figure presents the number of integral partitions required to bring the displacement evaluation errors to a level just below $0.1 \%$ (based on Equations (5) and (6)). This was calculated for different offset radii in the range $a^{+}$to $25 a$. Calculation results for $u_{z}^{0}$ are shown in Figure 5a and calculation results for $u_{r}^{0}$ are displayed in Figure 5b. All curves in the figure represent upper error envelope values. Efficiency levels for the standard evaluation method (Equations (3) and (4)) are plotted with dash-dotted lines. Efficiency levels of the proposed method (Equations (20) and (21)) are plotted with solid lines. Finally, the 
special case of the proposed method, wherein the polynomial coefficients in Equations (14) and (15) are all taken as zero, are represented by dashed lines. Because the proposed method consistently and independently of the radial offset requires less partitions, and because all curves represent a similar evaluation accuracy, it is deemed robustly superior.

\section{Summary and Remarks}

The core formulation of layered elastic theory consists of semi-infinite integrals with oscillating and computationally expensive integrands. The accepted numerical method for evaluating these integrals involves partitioning the domain of integration into a finite number of intervals defined by the integrand roots, and then integrating over these finite intervals with usual numerical techniques such as Gauss quadrature. Thus, the overall computational effort is essentially governed by the required number of partitions to achieve a given accuracy.

This paper proposed a new method for expediting surface displacement reevaluations in layered elastic systems. The particular case of surface responses is considered most computationally demanding as it is associated with slow decay of the integrands and therefore requires a large number of partitions. At the same time, it is also most relevant in the interpretation of measurements collected by emerging pavement evaluation devices. The proposed method was based on manipulating the original semiinfinite integrals prior to numerical integration by subtracting and then adding carefully selected auxiliary functions to the integrands (see Equations (10) and (11)). The auxiliary functions were formulated based on an analytic investigation of the integrand's behavior without limitation to certain structural types, i.e. without restrictions on the number of layers and corresponding parameter values. Overall, the suggested manipulation resulted in new integrals that were mathematically identical to the original integrals but more efficient to compute. Consequently, the approach is deemed robust and applicable to any layered elastic system. It is consistently more efficient than the standard evaluation method, requiring less partitions to achieve the same level of accuracy (as long as $z_{1} / H>1.5 \%$, refer to Figure 1). Moreover, it is especially suited for inverse analysis situations, wherein system responses at several predefined locations must be recomputed accurately and efficiently with new layer properties. 
Some directions for advancing this work include: (i) combining the proposed formulation with established techniques such as extrapolation (Maina and Matsui 2005), and integrand interpolation (Khazanovich and Wang 2007). This will confine the solution to a certain class of layered elastic problems in exchange for additional improvement in computational efficiency; (ii) varying the number of Gauss points employed in evaluating the finite integrals based, e.g., on interval size or some adaptive technique. So doing will further minimize the number of expensive integrand evaluations; (iii) developing new Accelerator functions $W_{z}$ and $W_{r}$ (see Equations (8) and (9)) that better match the behavior of $F_{z}$ and $F_{r}$ across a wider range of $m$ values and not only as $m \rightarrow \infty$; and (iv) combining the proposed method with the interpolation ideas in Pan et al. (2007), to further elevate computational efficiency whenever a very large number of surface solutions is sought.

\section{References}

AASHTO (2008), "Mechanistic-Empirical Pavement Design Guide - A Manual of Practice (Interim Edition).

ASTM D4695-03 (2015a), "Standard guide for general pavement deflection measurements," ASTM International, West Conshohocken, PA.

ASTM D4694-09 (2015b), "Standard test method for deflections with a falling-weighttype impulse load device," ASTM International, West Conshohocken, PA.

Abramowitz, M. (1954). "On the practical evaluation of integrals," Journal of the Society for Industrial and Applied Mathematics 2(1), pp. 20-35.

Andersen, S., Levenberg, E., and Andersen, M.B. (2017), "Inferring pavement layer properties from a moving measurement platform," Proceedings of the 10th International Conference on the Bearing Capacity of Roads, Railways and Airfields, Loizos, A., AlQadi, I., and Scarpas, T. (eds.), CRC Press, p. 394.

Chen, W.T. (1971). "Computation of stresses and displacements in a layered elastic medium,” International Journal of Engineering Science 9(9), pp. 775-800.

Cornille, P. (1972). "Computation of Hankel transforms," Society for Industrial and Applied Mathematics (SIAM) Review 14(2), pp. 278-285.

Dalla Valle, P. and Thom, N. (2016). "Improvement to method of equivalent thicknesses (MET) for calculation of critical strains for flexible pavements," International Journal of Pavement Engineering, DOI: 10.1080/10298436.2016.1238698.

Erlingsson, S. and Ahmed, A.W. (2013). "Fast layered elastic response program for the analysis of flexible pavement structures," Road Materials and Pavement Design 14(1), pp. 196-210.

Flintsch, G., Katicha, S., Bryce, J., Ferne, B., Nell, S., and Diefenderfer, B. (2013). "Assessment of continuous pavement deflection measuring technologies," Report S2R06F-RW-1, Transportation Research Board, Washington, D.C. 
Hillion, P. and Nurdin, G. (1977). "Integration of highly oscillatory functions," Journal of Computational Physics 23(1), pp. 74-81.

Huang, Y.H. (2004). Pavement Analysis and Design, Second Edition, Prentice-Hall, New Jersey.

Khazanovich, L. and Wang, Q. (2007). "MnLayer: high-performance layered elastic analysis program," Transportation Research Record 2037, pp. 63-75.

Liu, G.R. and Han, X. (2003). "Computational inverse Techniques in nondestructive evaluation," CRC Press, pp. 592.

Longman, I.M. (1956). "Note on a method for computing infinite integrals of oscillatory functions," Mathematical Proceedings of the Cambridge Philosophical Society 52(4), pp. 764-768.

Lucas, S.K. (1995). "Evaluation infinite integrals involving products of Bessel functions of arbitrary order," Journal of Computational and Applied Mathematics 64(3), pp. 269282.

Lucas, S.K. and Stone, H.A. (1995). "Evaluating infinite integrals involving Bessel functions of arbitrary order," Journal of Computational and Applied Mathematics 64(3), pp. 217-231.

Maina, J. and Matsui, K. (2005). "Elastic multi-layered analysis using DE-integration," Publications of the Research Institute for Mathematical Sciences 41(4), pp. 853-867.

Madsen, S.S. and Levenberg, E. (2017). "Dynamic backcalculation with different loadtime histories," Road Materials and Pavement Design, Road Materials and Pavement Design, DOI: 10.1080/14680629.2017.1307263.

Pan, E. (1997). "Static Green's functions in multilayered half spaces," Applied Mathematical Modeling 21(8), pp. 509-521.

Pan, E., Bevis, M., Han, F., Zhou, H., and Zhu, R. (2007). "Surface deformation due to loading of a layered elastic half-space: a rapid numerical kernel based on a circular loading element," Geophysical Journal International, Vol. 171(1), pp. 11-24.

Pedersen, L., Hjorth, P.G., and Knudsen, K. (2013). "Viscoelastic modelling of road deflections for use with the Traffic Speed Deflectometer. Thesis (PhD). Report IMMPHD-2013; No. 310, Technical University of Denmark (DTU), Kgs. Lyngby, Denmark.

Peutz, M.G.F, Van Kempen, H.P.M., and Jones, A. (1968). "Layered systems under normal surface loads," Highway Research Record 228, pp. 34-45.

Rada, G.R., Nazarian, S., Visintine, B.A., Siddharthan, R.V., and Sivaneswaran, N. (2015). "Use of high-speed deflection devices in network-level PMS applications: are we ready?" Paper presented at the 9th International Conference on Managing Pavement Assets, Alexandria, VA.

Sugihara, M. (1987). "Methods of numerical integration of oscillatory functions by the DE-formula with the Richardson extrapolation," Journal of Computational and Applied Mathematics 17(1-2), pp. 47-68.

US Army Corps of Engineers (2010). "PCASE 2.09.05 Desktop Software," Transportation Systems Center \& Engineering Research and Development Center, https://transportation.erdc.dren.mil/pcase/software.aspx (accessed: July 13, 2017).

Ullidtz, P. (1987). Pavement analysis, Elsevier, Amsterdam. 
Ullidtz, P., Harvey, J., Basheer, I., Jones, D., Wu, R., Lea, J., and Lu, Q. (2010). “CalME: mechanistic empirical design program for flexible pavement rehabilitation," Transportation Research Record 2153, pp. 143-152.

Wang, D., Roesler, J.R., and Guo, D.Z. (2010). "Innovative algorithm to solve axisymmetric displacement and stress fields in multilayered pavement systems," Journal of Transportation Engineering 137(4), pp. 287-295.

Zhou, F., Fernando, E., and Scullion, T. (2010). "Development, calibration, and validation of performance prediction models for the Texas M-E flexible pavement design system," Report FHWA/TX-10/0-5798-2, Texas Transportation Institute, College Station, Texas.

Zhao, Y., Zhou, C., Zeng, W., and Ni, Y. (2015). "Accurate determination of near-surface responses of asphalt pavements," Road Materials and Pavement Design 16(1), pp. 186199. 
Table 1. Layer thicknesses and elastic properties (see Figure 1).

\begin{tabular}{cccc}
\hline$n[-]$ & $z_{n}-z_{n-1}[\mathrm{~mm}]$ & $E_{n}[\mathrm{MPa}]$ & $v_{n}[-]$ \\
\hline 1 & 150 & 5000 & 0.30 \\
2 & 200 & 400 & 0.35 \\
3 & 400 & 200 & 0.35 \\
4 & $\infty$ & 70 & 0.40 \\
\hline
\end{tabular}

